\title{
Laser Process for Selective Emitter Silicon Solar Cells
}

\author{
G. Poulain, ${ }^{1}$ D. Blanc, ${ }^{1}$ A. Focsa, ${ }^{1}$ B. Bazer-Bachi, ${ }^{1}$ M. Gauthier, ${ }^{2}$ B. Semmache, ${ }^{3}$ Y. Pellegrin, ${ }^{3}$ \\ N. Le Quang, ${ }^{2}$ and M. Lemiti ${ }^{1}$
}

${ }^{1}$ Institut des Nanotechnologies de Lyon INL-UMR5270, CNRS, INSA de Lyon, Université de Lyon, 69621 Villeurbanne, France

${ }^{2}$ Photowatt International S.A.S., 33 rue Saint Honoré, 38300 Bourgoin-Jallieu, France

${ }^{3}$ SEMCO Engineering, 625, rue de la Croix Verte-Parc Euromédecine, 34196 Montpellier Cedex 5, France

Correspondence should be addressed to D. Blanc, daniele.blanc@insa-lyon.fr

Received 1 September 2011; Revised 7 December 2011; Accepted 15 December 2011

Academic Editor: Junsin Yi

Copyright ( 2012 G. Poulain et al. This is an open access article distributed under the Creative Commons Attribution License, which permits unrestricted use, distribution, and reproduction in any medium, provided the original work is properly cited.

\begin{abstract}
Selective emitter solar cells can provide a significant increase in conversion efficiency. However current approaches need many technological steps and alignment procedures. This paper reports on a preliminary attempt to reduce the number of processing steps and therefore the cost of selective emitter cells. In the developed procedure, a phosphorous glass covered with silicon nitride acts as the doping source. A laser is used to open locally the antireflection coating and at the same time achieve local phosphorus diffusion. In this process the standard chemical etching of the phosphorous glass is avoided. Sheet resistance variation from $100 \Omega$ /sq to $40 \Omega$ /sq is demonstrated with a nanosecond UV laser. Numerical simulation of the laser-matter interaction is discussed to understand the dopant diffusion efficiency. Preliminary solar cells results show a $0.5 \%$ improvement compared with a homogeneous emitter structure.
\end{abstract}

\section{Introduction}

Selective emitter technology can provide a significant increase in solar cell efficiency, but today most of the approaches need many processing steps and alignment procedure [1]. In this context, laser processing provides a good opportunity to achieve such a structure with a minimum number of technological steps [2-5] and without alignment when coupled with front side electrochemical metallisation [6]. Recent results have shown that around a $0.5 \%$ increase in solar cell efficiency could be obtained when using phosphosilicate glass (PSG) as a source of dopants to form highly doped areas on the emitter side according to Process A in Figure 1. In the perspective of a complete self-aligned process, a reduced number of technological steps is proposed to form selective emitters on the front surface of p-type silicon wafers (Figure 1, Process B). In this process, a homogenous emitter is formed by thermal diffusion of phosphorous. The resulting PSG layer is covered with silicon nitride $\left(\mathrm{SiN}_{x}\right)$ for passivation and antireflection purposes. Ablation of the $\mathrm{SiN}_{x}$ coating and dopant diffusion are then performed simultaneously with a pulsed laser before metallisation. Wet chemical etching of the PSG layer is therefore avoided and a self-aligned metallisation process can be developed with electrochemical deposition techniques. Numerical simulation of laser-matter interaction is presented to explain the heat-assisted diffusion mechanism and is compared to sheet resistance measurements. Laboratory scale solar cells are fabricated to compare homogeneous emitter and selective emitter structures. Spectral response and $I-V$ measurements are used to characterise the solar cells.

\section{Numerical Simulation of the Laser-Matter Interaction}

Melting, evaporation, and dopant diffusion are the main mechanisms that result from the laser-matter interaction. In a nanosecond regime, if the optical and thermal penetration depths are much smaller than the diameter of the incident laser beam on the surface, the thermal effects can be described by a two-dimensional heat-transfer equation [7]. The diffusion process can be estimated by solving at the same time Fick's second law. To estimate the impact of the 


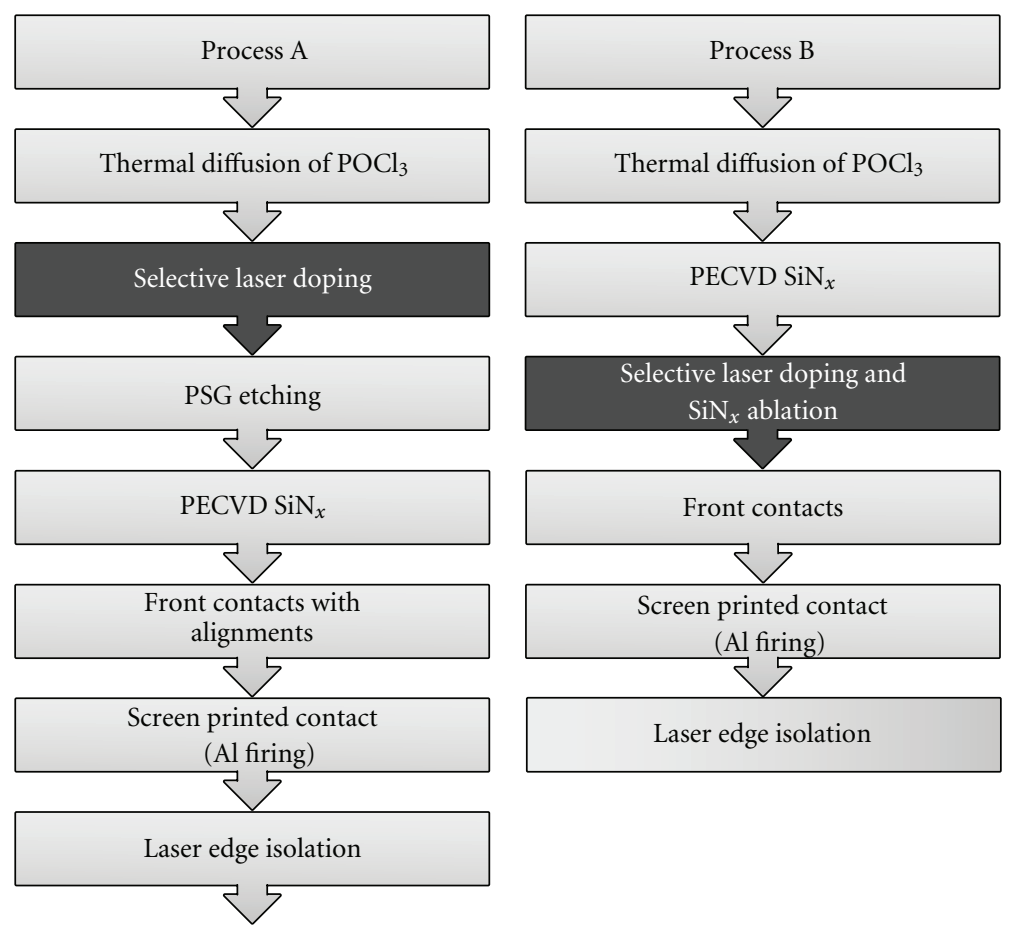

Figure 1: Processes for selective emitter solar cells with (Process A) and without (Process B) chemical etching and alignment steps.

laser parameters on the emitter formation, the finite element software COMSOL was used to solve these two equations [8]:

$$
\begin{gathered}
\rho(T) C_{p}(T) \frac{\partial T}{\partial t}=\nabla\left[k_{\mathrm{th}}(T) \nabla T\right]+Q \\
\frac{\partial c}{\partial t}=\nabla[D(T) \nabla c]
\end{gathered}
$$

$\rho(T)$ is the material density, $C_{p}(T)$ the specific heat capacity, $T$ the temperature, $t$ the time, $k_{\text {th }}$ the thermal conductivity, $Q$ the heat source in the volume due to the absorbed laser power, $c$ the phosphorous concentration in silicon, and $D(T)$ the diffusion coefficient of the phosphorous atoms in silicon. The heat source term $\mathrm{Q}$ in the heat-transfer equation corresponds to the absorbed laser power and can be written as follows:

$$
Q=(1-R(T)) \alpha(T) P_{\text {in }}(x, t) I(y) .
$$

$\alpha(T)$ is the material absorption coefficient, $R(T)$ the surface reflectivity, $P_{\text {in }}(x, t)$ the incident laser power, and $I(y)$ the relative intensity given by the Beer-Lambert law. The beam is considered to have a Gaussian shape in time and space:

$$
P_{\text {in }}(x, t)=P_{0} \exp \left\{-\left(\frac{t-t_{0}}{\tau / 2}\right)^{2}\right\} \exp \left\{-\left(\frac{x}{r}\right)^{2}\right\} .
$$

$P_{0}$ is the peak power of the laser beam, $t_{0}$ the time shift, $\tau$ the pulse duration, and $r$ the beam radius at half height.

Temperature dependence of the physical properties of the materials was taken into account. The smoothed Heaviside function (flc2hs) implemented in COMSOL was used to describe the abrupt changes of material coefficients with temperatures. Latent heat of fusion was taken into account by reducing the heat-source term for the temperatures above fusion by the quantity $E_{\mathrm{lm}} \cdot \rho / \tau$, where $E_{\mathrm{lm}}$ is the latent fusion heat, $\rho$ the density and $\tau$ the pulse duration. In a first approximation, the texturation of the surface was not taken into account and a plan of symmetry was considered in order to minimize the calculation time. The absorption coefficient of silicon at $355 \mathrm{~nm}$ is around $10^{8} \mathrm{~m}^{-1}$ corresponding to a penetration depth around $10 \mathrm{~nm}$. As a consequence, the minimum element size for calculation at the silicon surface was $1 \mathrm{~nm}$. The substrate was divided in different areas in order to have a finer meshing under the irradiated area. The time parameter varied from 0 to $60 \mathrm{~ns}$ with a step of $1 \mathrm{~ns}$ and $0.1 \mathrm{~ns}$ during the laser pulse (from $10 \mathrm{~ns}$ to $40 \mathrm{~ns}$ ). The sample geometry is represented in Figure 2.

For a better understanding of the role of each layer, temperature at the surface of silicon was calculated as a function of the laser fluence (Figure 3) for the different interfaces $\mathrm{Si} /$ air, Si/PSG/air, and Si/PSG/SiN $\mathrm{S}_{x}$ /air. Temperature variation at the silicon surface can be explained by the thermal properties of the layers (mostly the thermal conductivity) and by the different reflection coefficient $R$ of the surfaces. Laser doping efficiency is dependent on the temperature reached in silicon as the substrate has to be melted to allow efficient phosphorous diffusion into the junction. Indeed, the significant increase of the diffusion coefficient in the liquid state silicon $\left(\times 10^{7}\right)$ permits phosphorus diffusion during the short time of laser-matter interaction (tens of nanoseconds). As shown in Figure 3, the structure Si/PSG/SiN $x /$ air needs 


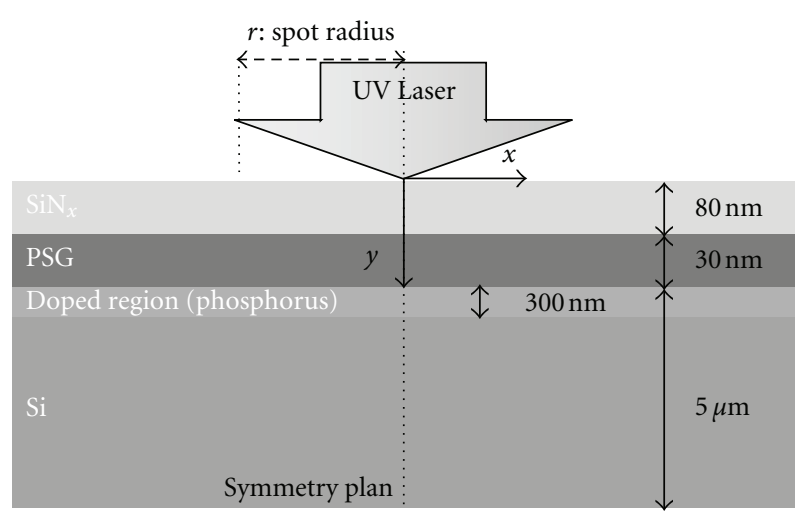

FIgURE 2: Sample geometry.

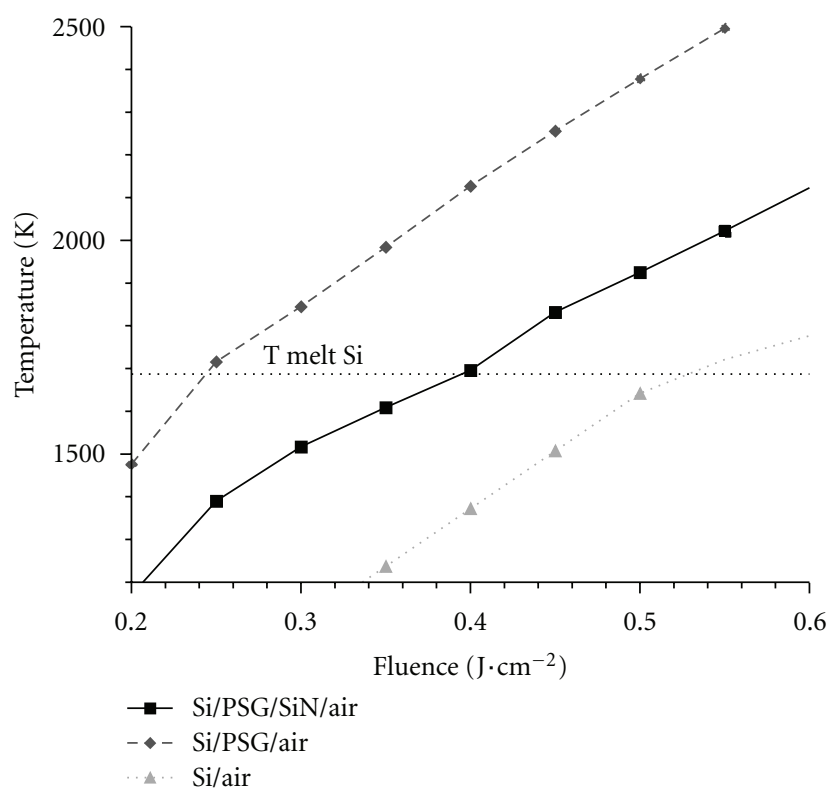

Figure 3: Simulated temperature versus laser fluence for different interfaces (Si/air, Si/PSG/air, Si/PSG/SiN/air).

more laser energy than the structure Si/PSG/air to melt the silicon substrate. Therefore a good control of the laser parameters is necessary to avoid heat-induced damages to the cell junction.

\section{Experimental}

Monocrystalline $\mathrm{Cz}$ silicon wafers with textured surfaces were used. Substrates were p-type with a thickness of $250 \mu \mathrm{m}$ and a resistivity of $1 \Omega \cdot \mathrm{cm}$. Homogeneous n-doped emitters with sheet resistance of $80 \Omega /$ sq and $100 \Omega$ /sq corresponding to surface doping concentration of $3.5 \times 10^{19} \mathrm{~cm}^{-3}$ and $2 \times 10^{19} \mathrm{~cm}^{-3}$, respectively, were fabricated by low-pressure thermal diffusion of $\mathrm{POCl}_{3}$ using the Lydop technology. The PSG layer formed at the wafer surface during thermal diffusion was preserved for use as a doping source during the laser-assisted diffusion process on the $100 \Omega$ /sq samples. The $80 \Omega /$ sq were kept as homogeneous emitters for comparison.
An $80 \mathrm{~nm}$ thick $\mathrm{SiN}_{x}$ layer was deposited over the PSG layer by conventional PECVD process for passivation and antireflection purposes. A frequency tripled Nd:YAG laser from ROFIN with a wavelength of $355 \mathrm{~nm}$ and a pulse duration of $10 \mathrm{~ns}$ was used for heat-assisted diffusion.

Simples test structures were fabricated to optimise the laser parameters. Test structures were characterised by optical microscopy, 4-point probe equipment, Transmission Line Method (TLM), and Quasi-Steady-State Photoconductance. Laboratory scale solar cells were characterised by illuminated $I-V$ and spectral response measurements.

3.1. Sheet Resistance Measurements. Figure 4 shows the typical evolution of the sheet resistance with laser fluence and pulse overlap measured on samples with a $100 \Omega /$ sq initial emitter. Complete ablation of $\mathrm{SiN}_{x}$ without debris was observed between $0.4 \mathrm{~J} \cdot \mathrm{cm}^{-2}$ and $0.7 \mathrm{~J} \cdot \mathrm{cm}^{-2}$. As expected from the simulation, laser-assisted doping was more efficient at low fluence for samples with PSG only. A decrease of the sheet resistance was measured at low fluence $\left(0.25 \mathrm{~J} \cdot \mathrm{cm}^{-2}\right)$ in PSG-capped silicon while no laser doping was measured for fluence under $0.4 \mathrm{~J} \cdot \mathrm{cm}^{-2}$ in PSG/SiN ${ }_{x}$-capped silicon. Moreover, $0.2 \mathrm{~J} \cdot \mathrm{cm}^{-2}$ and $50 \%$ overlap was sufficient to reach $65 \Omega /$ sq for a PSG-capped silicon while around $0.45 \mathrm{~J} \cdot \mathrm{cm}^{-2}$ and $50 \%$ overlap was necessary to reach the same doping level for a PSG/SiN ${ }_{x}$-capped silicon. On the other hand, at high fluence the minimum sheet resistance was lower for PSG/SiN $x_{x}$-capped silicon $(20 \Omega /$ sq) than for PSG-capped silicon $(30 \Omega / s q)$.

3.2. Passivation Properties. The influence of the PSG layer on the carrier lifetime was studied on different samples by transient decay photoconductance. FZ wafers with a thickness of $400 \mu \mathrm{m}$ and a resistivity of $800 \Omega \cdot \mathrm{cm}$ were used for thermal diffusion followed by a standard $\mathrm{SiN}_{x}$ deposition on both surfaces. The PSG layer was etched on one sample before $\mathrm{SiN}_{x}$ deposition. FZ samples with or without a PSG layer under the $\operatorname{SiN}_{x}$ coating showed similar lifetime around $200 \mu \mathrm{s}$ for a $60 \Omega / \mathrm{sq}$ emitter. A similar procedure was applied to a lower quality multicrystalline silicon wafer with a thickness of $250 \mu \mathrm{m}$ and a resistivity of $1 \Omega \cdot \mathrm{cm}$. Samples with PSG, PSG/ $\operatorname{SiN}_{x}$, and $\mathrm{SiN}_{x}$ on both faces were fabricated. All showed a similar lifetime around $10 \mu \mathrm{s}$. These first observations on high and low quality silicon substrates are promising for practical use of PSG/ $\mathrm{SiN}_{x}$ as passivating bilayer.

3.3. Laboratory Scale Solar Cell Results. Selective emitter solar cells were made following a modified version of the Process $B$ presented in Figure 1. Front side metallization was made by $\mathrm{Ti} / \mathrm{Pd} / \mathrm{Ag}$ evaporation preceded by a photolithography step. Homogeneous emitter solar cells were also fabricated following the same procedure without any laser step and with a selective chemical etching of $\mathrm{SiN}_{x}$. Selective emitter cells were realised on substrate with an initial emitter of $100 \Omega /$ sq and homogeneous emitter cells with an initial emitter of $80 \Omega /$ sq. Laser parameters were chosen to reach a sheet resistance of $40 \Omega / \mathrm{sq}$ under the metal contacts of 


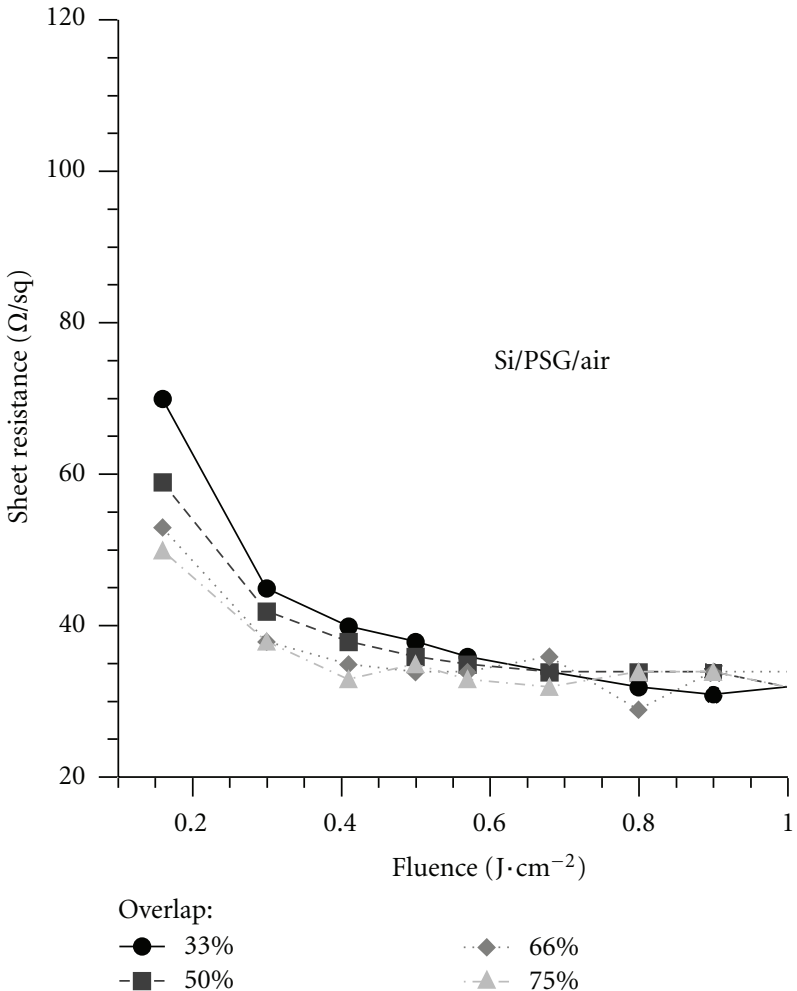

(a)

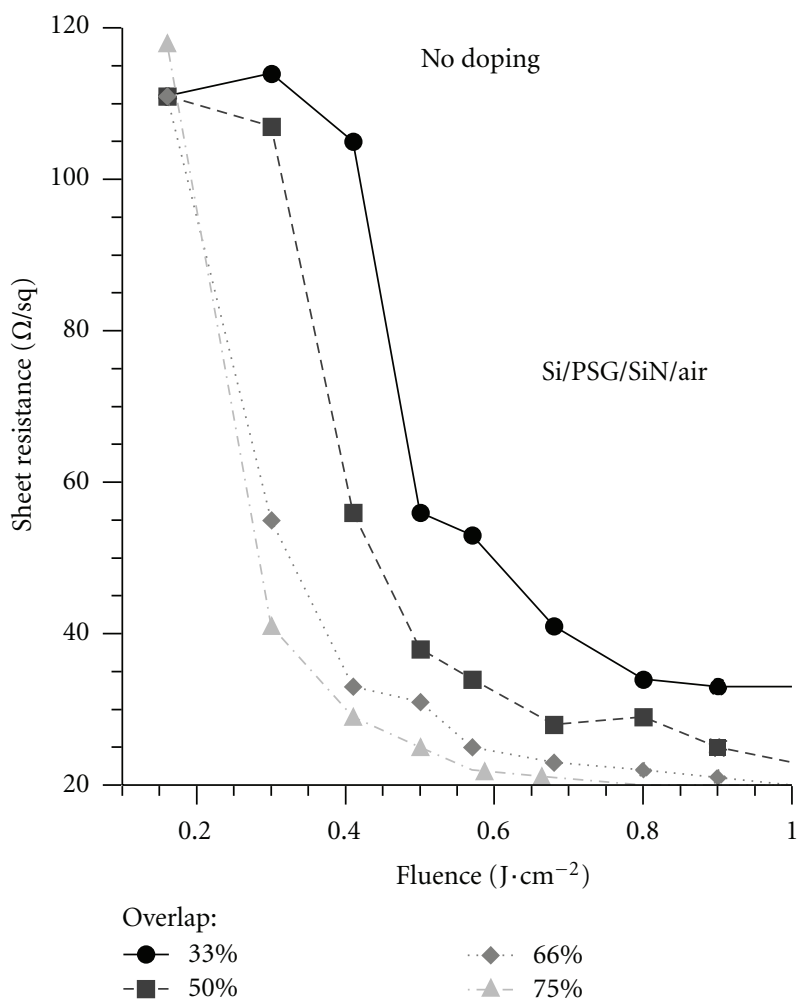

(b)

FIGURE 4: Sheet resistance evolution with laser fluence for $100 \Omega$ /sq initial emitters.

TABLE 1: Averaged cell parameters from illuminated $I-V$ results (HE stands for homogeneous emitter and SE selective emitter).

\begin{tabular}{lccccc}
\hline & $\begin{array}{c}\text { Initial } \\
\text { emitter }\end{array}$ & $\begin{array}{c}V_{\mathrm{oc}} \\
(\mathrm{mV})\end{array}$ & $\begin{array}{c}\text { Efficiency } \\
(\%)\end{array}$ & $\begin{array}{c}\mathrm{FF} \\
(\%)\end{array}$ & $\begin{array}{c}J_{\mathrm{sc}} \\
\left(\mathrm{mA} / \mathrm{cm}^{2}\right)\end{array}$ \\
\hline HE Average & $80 \Omega / \mathrm{sq}$ & 594 & 14.1 & 76.7 & 30.9 \\
SE Average & $100 \Omega / \mathrm{sq}$ & 604 & 14.6 & 75.4 & 32.1 \\
\hline
\end{tabular}

the selective emitter cells. Table 1 shows the average parameters extracted from the illuminated $I-V$ measurements with and without laser step. Nine cells $\left(4.1 \mathrm{~cm}^{2}\right)$ were measured for each process.

The selective emitter cells showed a $0.5 \%$ absolute increase in efficiency compared to the homogeneous emitter cells. The overall low efficiency of the solar cells was attributed to nonoptimized technological steps. Figure 5 shows an example of front contact metallisation misalignment that was observed in some of the cells. The lasertreated zone extends under the photoresist on the right hand side of the image. After metal deposition and chemical etching this area without passivation will be exposed to strong recombination. Lack of $\mathrm{Ti} / \mathrm{Pd} / \mathrm{Ag}$ metal sintering and nonoptimised antireflection properties of the PSG/SiN stack might also be responsible of the global loss of efficiency. For all cells, shunt resistance appears as the main cause

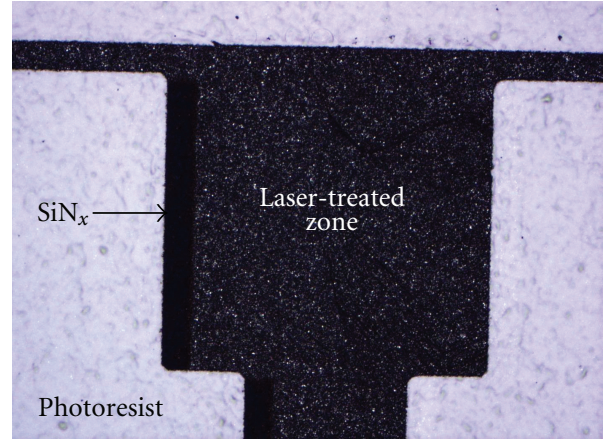

FIGURE 5: Image of the misalignment between the laser-treated zone and the metal contact area.

of parasitic resistive losses with an averaged value around $670 \Omega \cdot \mathrm{cm}^{2}$. Series resistance is reasonably low with an averaged value around $0.6 \Omega \cdot \mathrm{cm}^{2}$. The low values of $V_{\mathrm{oc}}$ are mainly attributed to recombination at the front surface.

Figure 6 shows the typical internal quantum efficiencies (IQEs) measured for homogeneous emitter (HE) and selective emitter (SE) cells. As expected, an increase of IQE is visible in the blue part of the spectrum for the selective emitter solar cells suggesting that the surface passivation is not degraded by the presence of the PSG layer at the surface. 


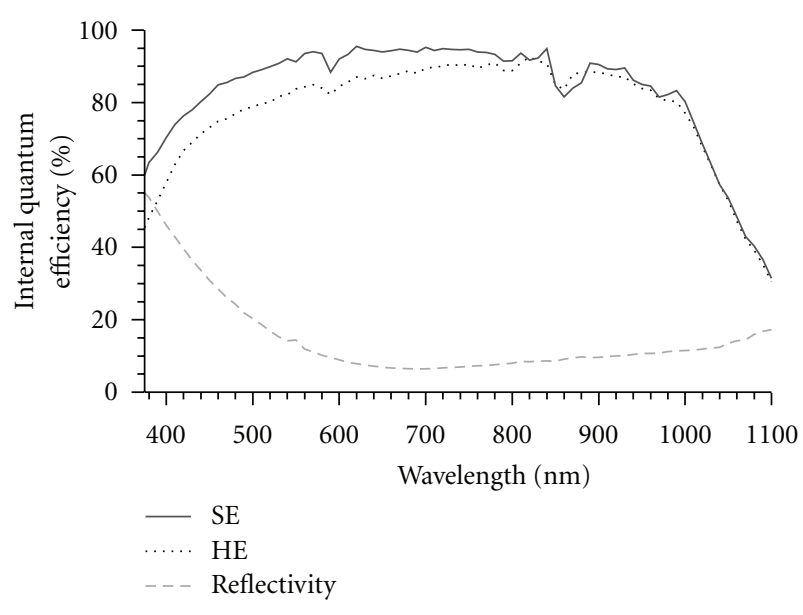

FIGURE 6: Internal quantum efficiency and reflectivity.

\section{Conclusion}

Selective emitter solar cells were fabricated with a reduced number of technological steps. Wet etching of the PSG was not necessary as this layer was preserved and covered by silicon nitride before laser-assisted diffusion. This process is potentially self-aligned if electrochemical front side metallisation is used. Preliminary results show reasonably good cell results with a $0.5 \%$ improvement compared with a homogeneous emitter structure. Several technological adjustments are foreseen on the antireflection and passivation properties of the PSG/SiN $x$ stack and on the front metal contact geometry to improve these first results. Long-term stability of the PSG layer has also to be investigated. Furthermore, numerical simulation of the laser-matter interaction is shown to be very useful to predict and understand the heatassisted diffusion mechanism.

\section{Acknowledgments}

This work was sponsored by the French Agency for Environment and Energy Management (ADEME) and Agence Nationale pour la Recherche (ANR).

\section{References}

[1] G. Hahn, "Status of selective emitter technology," in Proceedings of the 25th European PV Solar Energy Conference and Exhibition, pp. 1091-1096, Valencia, Spain, 2010.

[2] S. J. Eisele, T. C. Röder, J. R. Köhler, and J. H. Werner, “ $18.9 \%$ efficient full area laser doped silicon solar cell," Applied Physics Letters, vol. 95, no. 13, Article ID 133501, 3 pages, 2009.

[3] J. R. Köhler, P. Grabitz, S. J. Eisele, T. C. Roder, and J. H. Werner, "Laser doped selective emitters Yield 0.5\% efficiency gain," in Proceedings of the 24th European PV Solar Energy Conference and Exhibition, Hamburg, pp. 1847-1850, 2009.

[4] U. Jäger, M. Okanovic, M. Hörtheis, A. Grohe, and R. Preu, "Selective emitter by laser doping from phosphosilicate glass," in Proceedings of the 24th European PV Solar Energy Conference and Exhibition, pp. 1740-1743, 2009.
[5] B. Paviet-Salomon, S. Gall, S. Manuel, R. Monna, and A. Slaoui, "Laser doped selective emitter silicon solar Ccells," in Proceedings of the 25th European PV Solar Energy Conference and Exhibition, pp. 2179-2182, 2010.

[6] G. Poulain, C. Boulord, D. Blanc et al., "Direct laser printing for high efficiency silicon solar cells fabrication," Applied Surface Science, vol. 257, no. 12, pp. 5241-5244, 2011.

[7] J. Hermann, M. Benfarah, S. Bruneau et al., "Comparative investigation of solar cell thin film processing using nanosecond and femtosecond lasers," Journal of Physics D, vol. 39, no. 3, pp. 453-460, 2006.

[8] G. Poulain, D. Blanc, B. Semmache, Y. Pellegrin, and M. Lemiti, "Finite element simulation of laser-induced diffusion in silicon," Energy Procedia, vol. 8, pp. 587-591, 2011. 


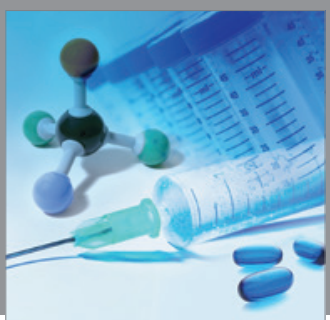

International Journal of

Medicinal Chemistry

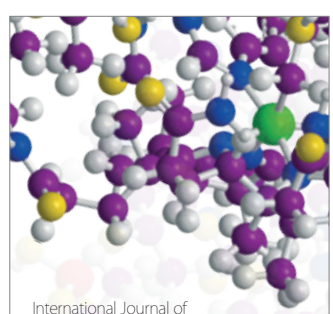

Carbohydrate Chemistry

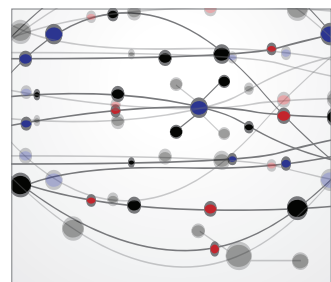

The Scientific World Journal
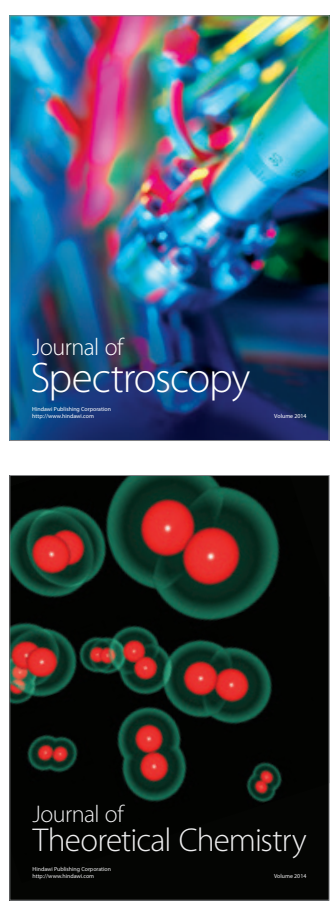
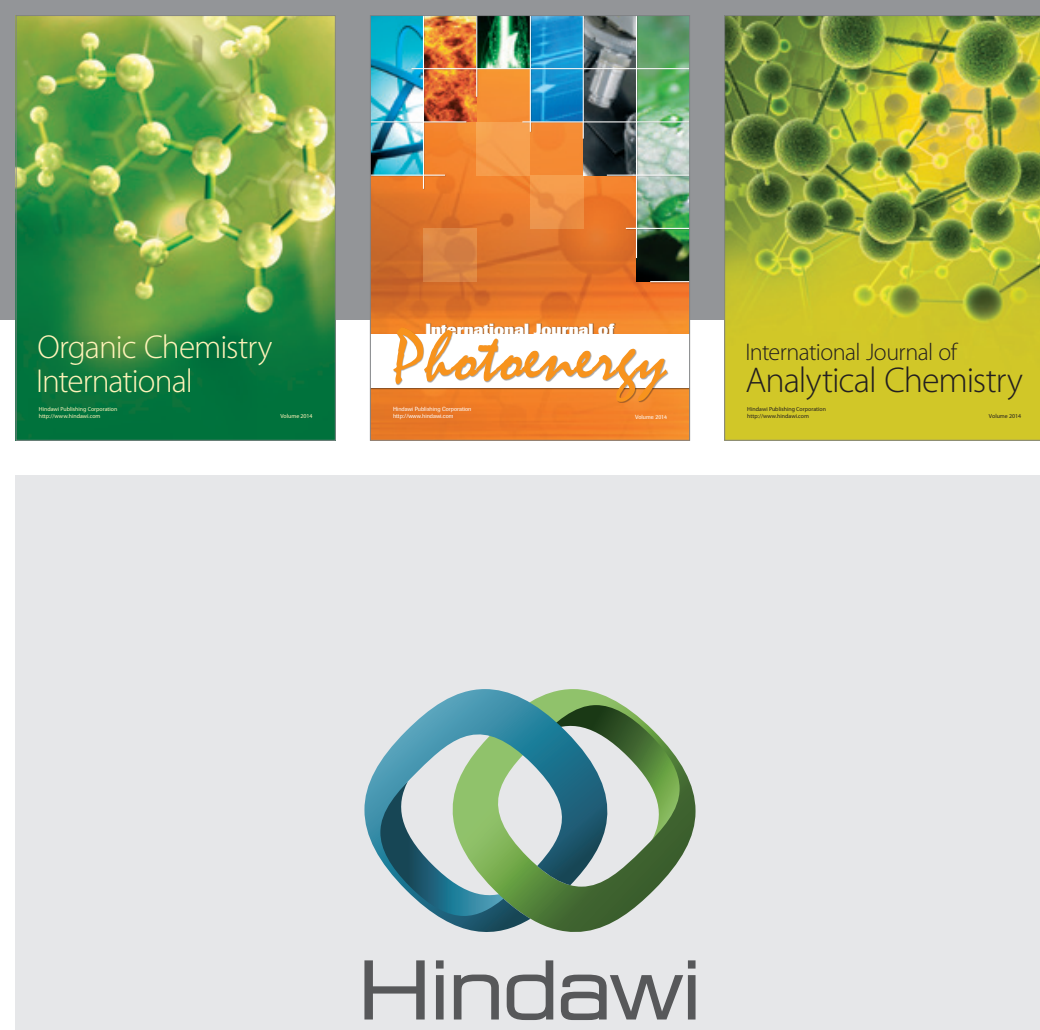

Submit your manuscripts at

http://www.hindawi.com
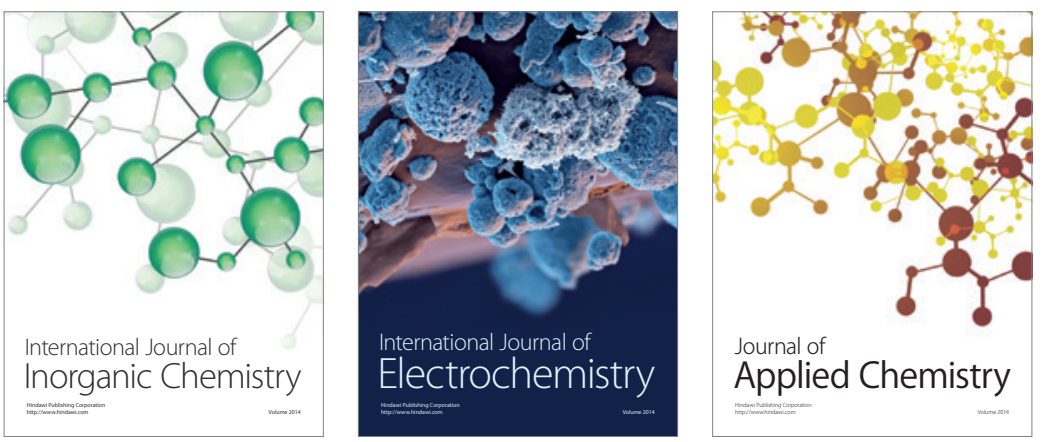

Journal of

Applied Chemistry
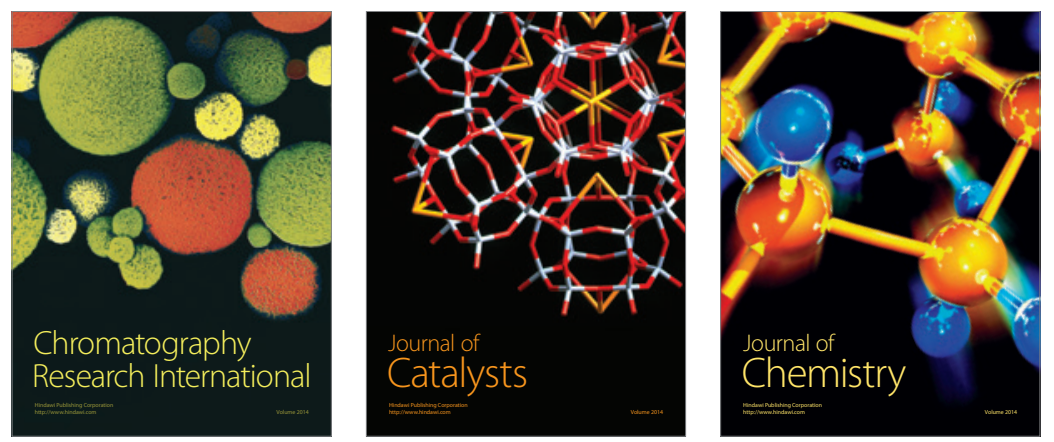
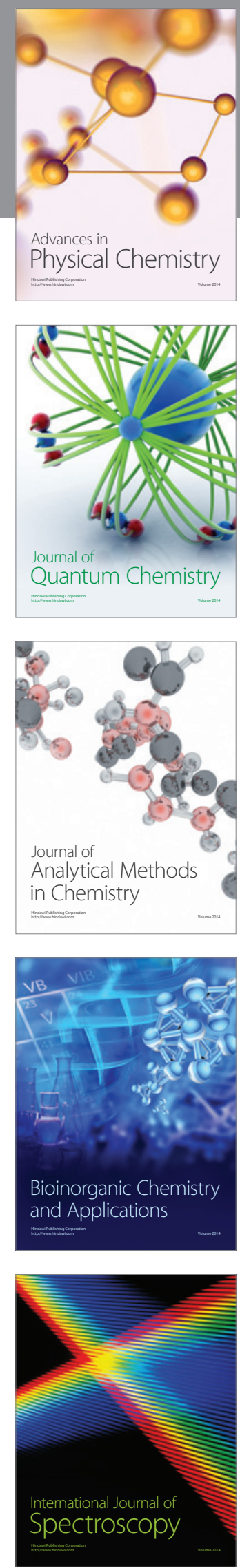